\title{
The Galactic mass distribution from the LAMOST Galactic spectroscopic surveys
}

\author{
Y. Huang ${ }^{1,4}$, X.-W. Liu ${ }^{1}$, H.-B. Yuan ${ }^{2}$, H.-W. Zhang ${ }^{1}$, M.-S. Xiang ${ }^{3,4}$, \\ B.-Q. Chen ${ }^{1,4}$ and C. Wang ${ }^{1}$ \\ ${ }^{1}$ Department of Astronomy, Peking University, Beijing 100871, China \\ email: yanghuang@pku.edu.cn \\ ${ }^{2}$ Department of Astronomy, Beijing Normal University, Beijing 100875, China \\ ${ }^{3}$ National Astronomical Observatories, Chinese Academy of Sciences, Beijing 100012, China \\ ${ }^{4}$ LAMOST Fellow (Supported by Special fund budgeted and administrated by CAMS)
}

\begin{abstract}
Using the data from the LAMOST Galactic spectroscopic surveys and some other surveys, we have started a series of work to measure the mass distribution of our Galaxy. As a result of the first-stage, we have constructed the Galactic rotation curve out to $100 \mathrm{kpc}$ and the Galactic escape velocity curve between 5 and $14 \mathrm{kpc}$. From the two curves, we have built parametrized mass models for our Galaxy, respectively. Both models yield a similar result for the Milky Way's virial mass: $\sim 0.9 \times 10^{12} \mathrm{M}_{\odot}$.
\end{abstract}

Keywords. Galaxy: fundamental parameters - Galaxy: kinematics and dynamics - dark matter

\section{The project: the Milky Way mass by LAMOST Survey}

Modelling the mass distribution of our Galaxy is one of the fundamental tasks of Galactic astronomy. An accurate measurement of the mass of the Milky Way (MW) is of vital importance for understanding the Galaxy formation and evolution. For example, the number of massive subhalos are highly decided by the virial mass of their host galaxies (e.g. Wang et al. 2012). Therefore, the key to understanding the so-called too big to fail problem (Boylan-Kolchin et al. 2011) for the MW is to accurately estimate the mass of our Galaxy. However, the current measurements of the MW mass are quite uncertain and the results could differ by a factor of 5 , from 0.5 to $2.5 \times 10^{12} \mathrm{M}_{\odot}$.

With the huge stellar spectra database collected by LAMOST (Cui et al. 2012), combined with other survey data (e.g. Gaia, SEGUE, APOGEE), we attempt to obtain an accurate measurement for the mass distribution of our Galaxy by a series of studies, including the Galactic rotation curve, the Galactic escape velocity curve, the cold stellar streams and the mass surface density. The Galactic rotation and escape velocity curves could be used to measure the overall mass of the MW, while the mass surface density could provide vital constraint on deriving the mass distribution of different Galactic components (i.e. the bulge, the disc and the dark matter). The study of cold stream could tell the shape information (e.g. spherical, oblate or prolate) of the dark matter. At present, we have finished the work of constructions of the Galactic rotation curve and the escape velocity curve and both studies give a similar result for the MW's virial mass: $0.9 \times 10^{12} \mathrm{M}_{\odot}$ (Huang et al. 2016; Huang et al. to be submitted). In what follows, we briefly introduce the constructions of the Galactic rotation curve and escape velocity curve, respectively.

First, using $\sim 6000$ primary red clump giants (PRCGs) in the outer disc selected from the LSS-GAC (Liu et al. 2014) and the SDSS-III/APOGEE survey, combined with 5700 

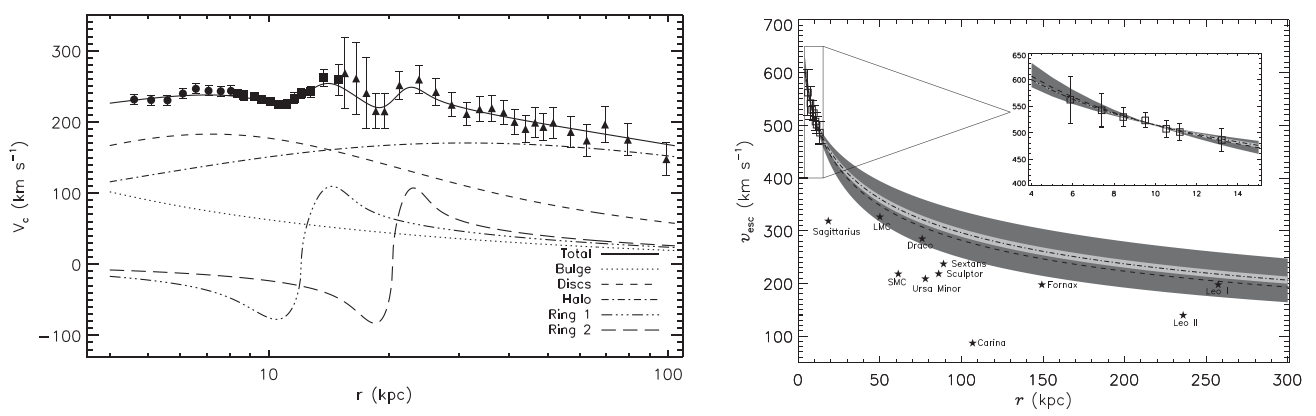

Figure 1. Left panel: Final combined rotation curve of the MW to $100 \mathrm{kpc}$ derived from $\mathrm{H}$ I data (dots), PRCGs (squares) and HKGs (triangles). Lines of different styles as labelled in the bottom-right corner of the diagram represent the best-fitting rotation curves contributions to the components of the Milky Way, with the solid line representing the sum of contributions from all the mass components. For details, please see Huang et al. (2016). Right panel: Galactic escape velocity $v_{\mathrm{esc}}$ as a function of Galactocentric radius $r$. Black boxes represent Galactic escape velocities newly derived from our sample of 527 high velocity halo stars for $r$ between 5 and $14 \mathrm{kpc}$. The dashed line and deep gray shade indicate our best-fit model and $1 \sigma$ uncertainty area without a prior on the local circular speed, while the dash-doted line and the shallow gray shade indicate those with a prior on the local circular speed. The inset in the top-right is to zoom in the region of the newly observed Galactic escape velocity curve for clarity. Black stars represent the total velocities of the named MW classical satellite galaxies. For details, please see Huang et al. (to be submitted).

halo K giants (HKGs) selected from the SDSS/SEGUE survey, we have constructed the rotation curve of the MW out to $\sim 100 \mathrm{kpc}$. The newly constructed rotation curve has a generally flat value of $240 \mathrm{~km} \mathrm{~s}^{-1}$ within $r$ of $25 \mathrm{kpc}$ and then decreases steadily to $150 \mathrm{~km} \mathrm{~s}^{-1}$ at $r \sim 100 \mathrm{kpc}$ (See the left panel of Fig. 1).

Secondly, using a sample of 527 high velocity halo stars $\left(\left|v_{r}\right| \geqslant 300 \mathrm{~km} \mathrm{~s}^{-1}\right.$ and $[\mathrm{Fe} / \mathrm{H}]<$ -1) selected from the LAMOST Galactic spectroscopic surveys, we have measured the Galactic escape velocities at $r$ between 5 and $14 \mathrm{kpc}$. The Galactic escape velocity at the solar position $v_{\text {esc }}\left(R_{0}\right)$ is found to $529 \pm 29 \mathrm{~km} \mathrm{~s}^{-1}$ (90 per cent confidence). As the right panel of Fig. 1 shows, the newly constructed Galactic escape velocity curve decreases steadily from $562 \mathrm{~km} \mathrm{~s}^{-1}$ at $r \sim 5.9 \mathrm{kpc}$ to $486 \mathrm{~km} \mathrm{~s}^{-1}$ at $r \sim 13.2 \mathrm{kpc}$.

Finally, from the constructed rotation and escape velocity curves, we have built parametrized mass models for our Galaxy, respectively. Both models yield a similar results of the virial mass of the MW's dark matter halo of $0.9 \pm 0.1 \times 10^{12} \mathrm{M}_{\odot}$. The local dark matter density is also found to be $\sim 0.008 \pm 0.001 \mathrm{M}_{\odot} \mathrm{pc}^{-3}$ from both models.

\section{Acknowledgements}

This work is supported by the National Key Basic Research Program of China 2014CB845700.

\section{References}

Boylan-Kolchin, M., Bullock, J. S., \& Kaplinghat, M. 2011, MNRAS, 415, L40

Cui, X.-Q., Zhao, Y.-H., Chu, Y.-Q., et al. 2012, RAA, 12, 1197

Huang, Y., Liu, X.-W., Yuan, H.-B., et al. 2016, MNRAS, 463, 2623

Liu X. -W., et al., 2014, in Feltzing S., Zhao G., Walton N., Whitelock P., eds, Proc. IAU Symp. 298, Setting the scene for Gaia and LAMOST, Cambridge University Press, pp. 310-321, preprint (arXiv: 1306.5376)

Wang, J., Frenk, C. S., Navarro, J. F., Gao, L., \& Sawala, T. 2012, MNRAS, 424, 2715 